\title{
Automatic Analysis of 3D Scans of Professional Athletes
}

\author{
Andrea GIACHETTI ${ }^{*}$, Francesco PISCITELLI ${ }^{2}$, Valentina CAVEDON ${ }^{2}$ \\ Chiara MILANESE ${ }^{2}$, Carlo ZANCANARO ${ }^{2}$ \\ ${ }^{1}$ Dpt. of Computer Science, University of Verona, Verona, Italy; ${ }^{2}$ Lab. of Anthropometry \& Body \\ Composition, Dpt. of Neurological and Movement Sciences, Verona, Italy
}

DOI: 10.15221/15.092 http://dx.doi.org/10.15221/15.092

\begin{abstract}
In this paper we present an analysis of body features of professional athletes performed using 3D body scanning with automatic processing and measurement of acquired 3D meshes and body composition data from dual-energy X-ray absorptiometry (DXA) acquisition. The aim of the work was to investigate whether professional male athletes practicing different sports show sport-specific features in terms of specific body dimensions and body composition. To perform the study, we collected 3D body scans and DXA scans of 211 players practicing basketball, soccer, golf, handball, rugb, volleyball as well as a control group of 38 physically active young adults.

A set of geometrical parameters were extracted automatically from the models exploiting a custom software tool based on body segmentation based curve skeleton analysis and symmetry based heuristics and previously applied with success to the analysis of body fat.

By measuring these body features from the scans, we could perform statistical analysis of their correlation with body composition parameters and also analyze differences among sports, in order to understand which features are more characterizing individual sports.

Furthermore, we checked if combinations of the selected feature measurements could possibly be characteristics of the disciplines and/or distinguish between professional athletes and physically active subjects, by visually analyzing the multidimensional feature space and testing automatic "athlete" or "discipline" labeling in a leave one out classification framework using different feature combinations and different classification methods. This allowed us to extract the most relevant features related to each different group.
\end{abstract}

\section{Introduction}

Over the past several years, much research has been focused on the identification of anthropometric and body composition variables able to discriminate athletes from different sports and their relative contribution; accordingly, understanding the sport-specific relationships between body dimensions and body composition is an issue in sports science. For example, recognizing predictor variables related to a performance criterion and/or allowing discrimination between different ability levels would be of help for coaches and strength and conditioning professionals [1,2,3,4]. For this reason, recent advance in digital anthropometry can be helpful in providing useful information to recover interesting insights related to these relationships.

While using traditional anthropometry the collection of relevant morphological measurement would be extremely time consuming (and operator-depending). Our research group recently developed a method to collect automatically a set of morphological measurements that has been successfully applied for the indirect estimation of body fat and to characterize human morphotypes [5].

In this work we use this tool, together with other acquisition methods (weight measurements, anthropometry and DXA scanning) to analyze the body features of different sets of professional athletes, trying to derive peculiar statistical characteristics of each group that can be considered distinctive of the specific sports or are likely to be changed by the specific activity.

\section{Materials and methods}

To perform the study, we collected 3D body scans and DXA scans of 211 players practicing basketball $(n=51)$, soccer $(n=89)$, golf $(n=9)$, handball $(n=22)$, rugby $(n=27)$, volleyball $(n=13)$ as well as a control group of 38 physically active young adults. Body scans were acquired with a Breuckmann Bodyscan, while DXA scan were obtained with a QDR Explorer W (Hologic, MA, USA). Both the devices are available at the Department of Neurologic and Movement Sciences, University of Verona.

Acquired models were post processed with a Meshlab [6] script to remove outliers and floor points, remeshed with the Poisson method in order to obtain watertight models, and simplified with the quadric edge collapse method in order to reduce their complexity and get a controlled number of faces (20K).

*andrea.giachetti@univr.it; +39 045 8027998; www.andreagiachetti.it 
On the resulting models, a software implementing the processing pipeline described in [5] has been applied. This software estimates a curve skeleton of the model, a stick figure encoding pose, roughly segment body regions (trunk, head, limbs) and estimate a set of anthropometric measures not depending on accurate landmarking. Examples of segmented meshes are represented in Figure 1.

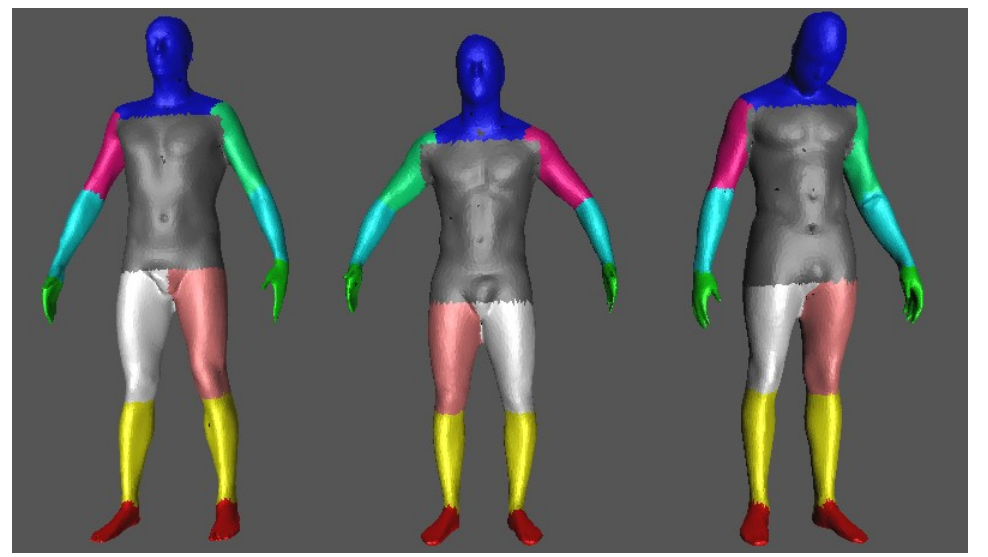

Figure 1: Example of segmented body scans of a professional basketball player (left), a professional rugby player (center), a professional golf player (right)

The list of the estimated values is the following: volume of the watertight mesh obtained after Poisson remeshing (VOL); Surface area of the watertight mesh obtained after Poisson remeshing (S); height measured as distance of the head tip from the floor $(\mathrm{H})$, volume of the segmented trunk (TVOL); maximal average trunk section radius (MAVR); maximal anteroposterior distance (MAX_AP); maximal trunk width (MAXW); maximal transversal size across the sections (MAXTW); minimal transversal size across the sections (MITW); maximal trunk section area (MTA); roundness 1 [20]: eccentricity ofan ellipse where the major axis is equal to the subjects' height and the minor axis equal to the transversal size at the maximal width (RND1); roundness 2, like RND1, but the minor axis equal to the transversal size at the minimal width (RND2); Maximal distance from border in the forearm (FA); maximal distance from border in the calf region (CA); maximal distance from border in the thigh region (TH). Many of these measure demonstrated to be stable in different acquisition and correlate well with body fat values estimated with the DXA scan.

We collected all these values for the selected meshes, together with the corresponding manual measurements (height, weight, BMI) and selected DXA values: trunk fat, trunk lean, trunk fat percentage, whole body fat, whole body lean, whole body fat percentage. In the following section we report some results of a data analysis performed on all these data.

\section{Data analysis}

\subsection{Differences between sports groups and control group}

As a first test, we analyzed differences of estimated values for the selected groups of professional athletes with respect to the control group. We performed t-tests comparing averages of the groups and found, as expected differences with high statistical significance for many parameters.

Tables 1, 2 and 3 show the $p$ values estimated for height/weight values, DXA and scanner based parameters, computed from the t values for the specific numbers of samples. As expected, differences between the groups and the reference set are often statistically significant, as bodies of professional players are bigger and fitter.

As expected, rugby players present a large difference with respect to the control group related to body size and also fat percentage. Basketball and volley players are quite different due to height, handball players are the most similar to the control group. However, there are also some specific features that are less expected and can be used to understand specific features of the group. Rugby players are the only group with a quite large difference in BMI wrt the control group. On the other hand handball and golf players are not relevantly different from the control group considering body size and weight. Volleyball and basketball players could be considered quite similar, but looking at some specific measurements they differ from the control group in specific ways (differences in forearm size are more evident for volley, for thigh size are more relevant for basketball). Golf players present a relevant trunk volume and trunk fat value. 


\begin{tabular}{|l|r|r|r|}
\cline { 2 - 4 } \multicolumn{1}{c|}{} & \multicolumn{1}{c|}{ WEIGHT } & \multicolumn{1}{c|}{ HEIGHT } & \multicolumn{1}{c|}{ BMI } \\
\hline volleyball & $3,22 \mathrm{E}-06$ & $1,28 \mathrm{E}-12$ & $2,48 \mathrm{E}-02$ \\
\hline soccer & $1,86 \mathrm{E}-04$ & $2,10 \mathrm{E}-08$ & $5,47 \mathrm{E}-01$ \\
\hline rugby & $6,31 \mathrm{E}-09$ & $4,46 \mathrm{E}-04$ & $1,05 \mathrm{E}-07$ \\
\hline handball & $1,29 \mathrm{E}-02$ & $3,45 \mathrm{E}-02$ & $4,38 \mathrm{E}-02$ \\
\hline golf & $1,45 \mathrm{E}-01$ & $2,68 \mathrm{E}-01$ & $2,76 \mathrm{E}-01$ \\
\hline basketball & $\mathbf{8 , 6 2 \mathrm { E } - 0 9}$ & $5,48 \mathrm{E}-14$ & $3,50 \mathrm{E}-01$ \\
\hline
\end{tabular}

Table 1: $P$ values of t-tests evaluating statistical relevance of differences of manually estimated values of specific sports groups and a reference group of healthy subjects. Highlighted values indicate values lower than $1 \%$

\begin{tabular}{|l|r|r|r|r|r|r|}
\cline { 2 - 7 } \multicolumn{1}{c|}{} & TRUNK_FAT & TRUNK_LEAN_1 & TRUNK_PFAT & WBTOT_FAT & WBTOT_LEAN_1 & WBTOT_PFAT \\
\hline volleyball & $6,27 \mathrm{E}-01$ & $2,00 \mathrm{E}-07$ & $8,89 \mathrm{E}-02$ & $7,72 \mathrm{E}-01$ & $8,20 \mathrm{E}-08$ & $5,25 \mathrm{E}-02$ \\
\hline soccer & $8,67 \mathrm{E}-02$ & $7,57 \mathrm{E}-08$ & $3,66 \mathrm{E}-04$ & $2,01 \mathrm{E}-02$ & $2,17 \mathrm{E}-09$ & $1,00 \mathrm{E}-05$ \\
\hline rugby & $1,46 \mathrm{E}-04$ & $4,74 \mathrm{E}-08$ & $2,04 \mathrm{E}-03$ & $2,23 \mathrm{E}-05$ & $1,05 \mathrm{E}-09$ & $1,60 \mathrm{E}-03$ \\
\hline handball & $1,06 \mathrm{E}-01$ & $3,30 \mathrm{E}-02$ & $1,76 \mathrm{E}-01$ & $3,07 \mathrm{E}-02$ & $1,91 \mathrm{E}-02$ & $6,87 \mathrm{E}-02$ \\
\hline golf & $1,69 \mathrm{E}-02$ & $9,65 \mathrm{E}-01$ & $1,58 \mathrm{E}-03$ & $1,35 \mathrm{E}-02$ & $9,33 \mathrm{E}-01$ & $1,63 \mathrm{E}-03$ \\
\hline basketball & $4,29 \mathrm{E}-01$ & $2,01 \mathrm{E}-08$ & $7,60 \mathrm{E}-01$ & $1,86 \mathrm{E}-01$ & $7,78 \mathrm{E}-11$ & $7,16 \mathrm{E}-01$ \\
\hline
\end{tabular}

Table 2: $P$ values of $t$-tests evaluating statistical relevance of differences of DXA values of specific sports groups and the reference group. Highlighted values indicate values lower than $1 \%$

\begin{tabular}{|c|c|c|c|c|c|c|c|c|c|c|c|c|c|c|}
\hline & VOL & $\mathrm{s}$ & $\mathrm{H}$ & TVOL & MAX_AP & MAXW & RND1 & RND2 & MAVR & MAXA & MITW & FA & CA & TH \\
\hline volleyball & $5,26 \mathrm{E}-06$ & $1,74 \mathrm{E}-08$ & $2,07 E-11$ & $8,33 \mathrm{E}-01$ & $5,44 \mathrm{E}-02$ & 1,17E-05 & $1,03 E-02$ & $3,84 \mathrm{E}-02$ & $6,15 \mathrm{E}-03$ & $2,72 \mathrm{E}-03$ & $1,39 E-01$ & 3,63E-03 & $2,34 \mathrm{E}-02$ & $1,64 \mathrm{E}-02$ \\
\hline soccer & $1,35 \mathrm{E}-02$ & $9,28 \mathrm{E}-04$ & $9,84 \mathrm{E}-06$ & $4,59 \mathrm{E}-01$ & $9,43 \mathrm{E}-01$ & $3,11 \mathrm{E}-01$ & $1,05 E-03$ & $5,25 \mathrm{E}-01$ & $1,60 \mathrm{E}-01$ & $9,12 \mathrm{E}-02$ & $1,82 \mathrm{E}-01$ & $5,01 \mathrm{E}-01$ & $8,91 \mathrm{E}-01$ & $3,84 \mathrm{E}-01$ \\
\hline rugby & $1,74 \mathrm{E}-08$ & $6,04 \mathrm{E}-08$ & $1,18 \mathrm{E}-03$ & $8,61 \mathrm{E}-01$ & $1,11 \mathrm{E}-06$ & $2,99 \mathrm{E}-07$ & $3,68 \mathrm{E}-04$ & $2,57 \mathrm{E}-05$ & $3,38 \mathrm{E}-08$ & $1,64 \mathrm{E}-08$ & $1,82 \mathrm{E}-06$ & $7,30 \mathrm{E}-04$ & 4,09E-04 & $5,22 \mathrm{E}-05$ \\
\hline handball & $1,44 \mathrm{E}-02$ & $4,26 \mathrm{E}-02$ & $1,29 \mathrm{E}-01$ & $5,04 \mathrm{E}-01$ & $4,43 E-02$ & $2,00 \mathrm{E}-03$ & $1,68 \mathrm{E}-02$ & $1,88 \mathrm{E}-03$ & $2,85 \mathrm{E}-02$ & $1,82 \mathrm{E}-02$ & $1,41 \mathrm{E}-03$ & $1,87 \mathrm{E}-01$ & $8,34 \mathrm{E}-01$ & $4,63 \mathrm{E}-02$ \\
\hline \begin{tabular}{|l|} 
golf \\
\end{tabular} & $1,64 \mathrm{E}-01$ & $3,56 \mathrm{E}-01$ & 4,99E-01 & $2,48 \mathrm{E}-03$ & $1,95 \mathrm{E}-01$ & $1,78 \mathrm{E}-01$ & 4,03E-01 & $1,83 \mathrm{E}-01$ & 1,19E-01 & $9,34 \mathrm{E}-02$ & $8,21 \mathrm{E}-02$ & $3,68 \mathrm{E}-01$ & $9,90 \mathrm{E}-01$ & $6,15 \mathrm{E}-01$ \\
\hline basketball & 4,49E-08 & $3,26 \mathrm{E}-10$ & $4,62 \mathrm{E}-12$ & $4,96 \mathrm{E}-01$ & $1,30 \mathrm{E}-02$ & $1,62 \mathrm{E}-04$ & $2,45 E-03$ & $9,69 \mathrm{E}-02$ & 1,14E-04 & $2,56 \mathrm{E}-04$ & 1,73E-05 & $8,35 \mathrm{E}-01$ & $6,66 \mathrm{E}-02$ & $3,40 \mathrm{E}-04$ \\
\hline
\end{tabular}

Table 3: $P$ values of t-tests evaluating statistical relevance of differences of automatically computed shape mesures of specific sports groups and the reference group. Highlighted values indicate values lower than $1 \%$

It must also be noted that measurements are correlated and that, for example, many differences in measurements are due only to different body sizes. We normalized a set of measurements depending on body size (MAX_AP,MAXW,MAXAVR, MAXA, MITW, FA, CA, TH) dividing values by body height or surface in order to obtain a dimensionless variable. Table 4 represents the $p$ values in t-tests comparing differences of these variables with respect to the control group. We see that here differences are significant at the $1 \%$ level less frequently, and specific values emerge as potential characteristics of groups (e.g. MAXW/H for basketball, rugby and soccer players).

\begin{tabular}{|l|r|r|r|r|r|r|r|r|}
\cline { 2 - 9 } \multicolumn{1}{c|}{} & \multicolumn{1}{c|}{ MAX_AP/H } & \multicolumn{1}{l|}{ MAXW/H } & \multicolumn{1}{l|}{ MAVR/H } & MAXA/S & MITW/H & FA/H & \multicolumn{1}{l|}{ CA/H } & TH/H \\
\hline volleyball & $1,85 \mathrm{E}-04$ & $1,37 \mathrm{E}-02$ & $1,92 \mathrm{E}-05$ & $6,39 \mathrm{E}-01$ & $8,61 \mathrm{E}-01$ & $8,71 \mathrm{E}-01$ & $5,04 \mathrm{E}-03$ & $1,53 \mathrm{E}-03$ \\
\hline soccer & $4,06 \mathrm{E}-03$ & $1,39 \mathrm{E}-03$ & $1,29 \mathrm{E}-02$ & $6,19 \mathrm{E}-01$ & $7,75 \mathrm{E}-01$ & $5,49 \mathrm{E}-01$ & $9,49 \mathrm{E}-03$ & $7,93 \mathrm{E}-03$ \\
\hline rugby & $2,65 \mathrm{E}-05$ & $3,48 \mathrm{E}-04$ & $5,66 \mathrm{E}-06$ & $2,93 \mathrm{E}-01$ & $1,06 \mathrm{E}-01$ & $8,89 \mathrm{E}-03$ & $2,23 \mathrm{E}-02$ & $2,38 \mathrm{E}-02$ \\
\hline handball & $1,20 \mathrm{E}-01$ & $1,42 \mathrm{E}-02$ & $1,11 \mathrm{E}-01$ & $2,49 \mathrm{E}-01$ & $3,03 \mathrm{E}-02$ & $3,78 \mathrm{E}-01$ & $5,48 \mathrm{E}-01$ & $1,35 \mathrm{E}-01$ \\
\hline golf & $2,47 \mathrm{E}-01$ & $3,19 \mathrm{E}-01$ & $1,59 \mathrm{E}-01$ & $7,24 \mathrm{E}-02$ & $1,22 \mathrm{E}-02$ & $5,28 \mathrm{E}-01$ & $7,44 \mathrm{E}-01$ & $3,97 \mathrm{E}-01$ \\
\hline basketball & $3,95 \mathrm{E}-01$ & $8,35 \mathrm{E}-03$ & $1,14 \mathrm{E}-01$ & $5,92 \mathrm{E}-01$ & $5,72 \mathrm{E}-01$ & $1,08 \mathrm{E}-02$ & $1,51 \mathrm{E}-03$ & $8,02 \mathrm{E}-03$ \\
\hline
\end{tabular}

Table 4: $P$ values of $t$-tests evaluating statistical relevance of differences of automatically computed shape measurements scaled by body size of specific sports groups and the reference group.

Highlighted values indicate values lower than $1 \%$

\subsection{Multivariate data visualization}

As it would be too complex and messy to analyze all the inter-classes differences, we decided then to use some information visualization tools to try to figure out the peculiar characteristics of the different groups. A way to try to evaluate differences between groups on multivariate data is to map features on a 2 (or) 3 dimensional space using Multidimensional Scaling [7]. With this technique, given as input the matrix giving dissimilarities between pairs of measured feature vectors, it is possible to obtain the 2-dimensional space such that the between-object distances are preserved as well as possible.

We relied on the implementation of classical MDS provided by the PrTools package [8].

Figure 2 shows the two dimensional plots representing all the subjects in the dataset in the mapped spaces obtained from Height/Weight/BMI (top-left), DXA values (top right), Full set of automatic measurements (bottom left) subset of scaled measurements (bottom right). As expected, in the first plot we can see sufficiently well separated most of the rugby players, and most of the volley and basketball players, placed in the same region, while other groups are mixed. The second plot reveals that golf players seem to be characterized by fat values. Plots based on geometrical measurements are less clear, but it must be considered that here the original space dimensionality is higher. 

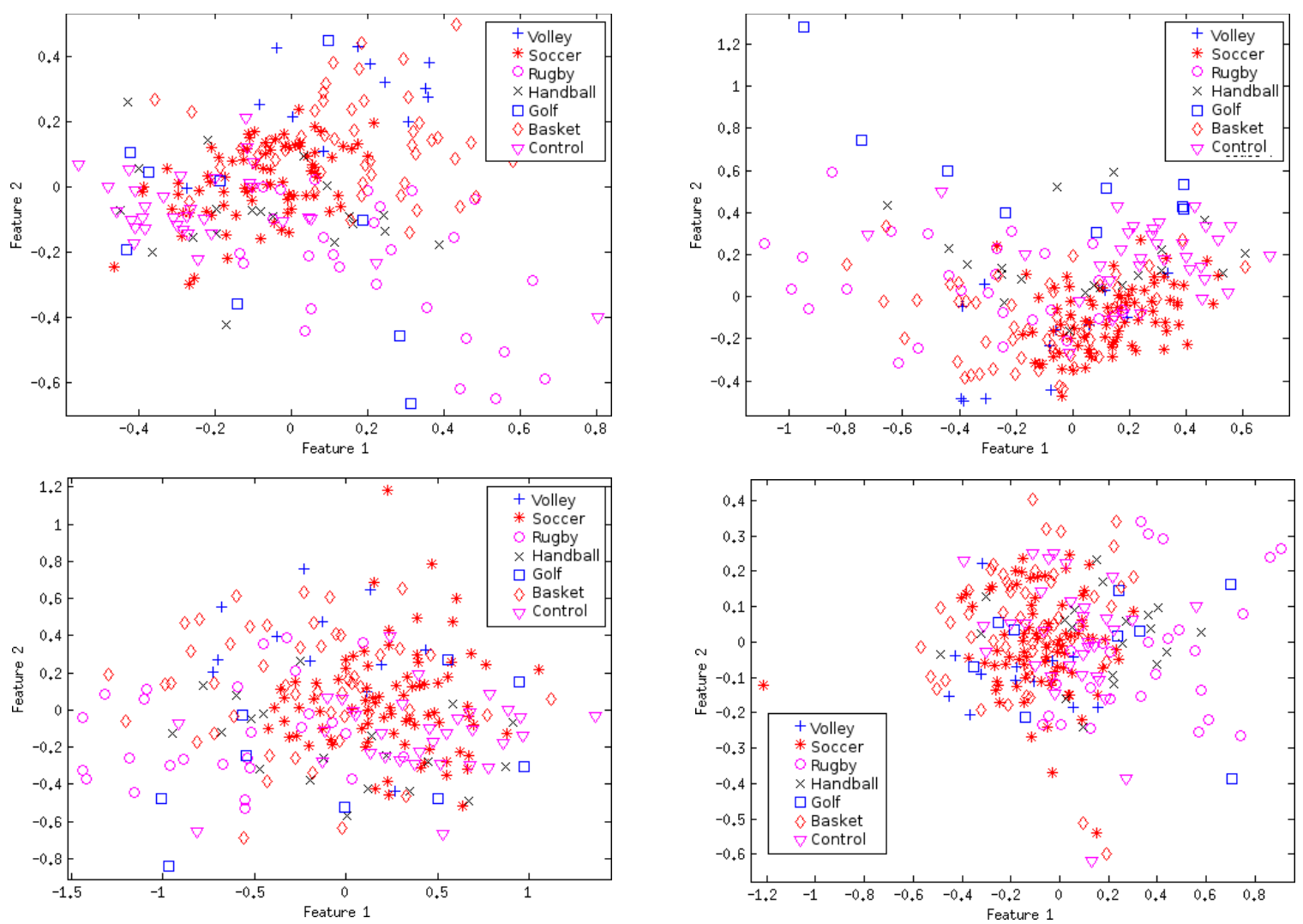

Figure 2: Scatter plots of feature spaces mapped onto 2-dimensions using Multidimensional scaling (MDS).

Top left: height/weight/BMI. Top right: DXA values space. Bottom left: full automatic measurement set. Bottom right: normalized subset of automatic measurements

To have a clearer idea of which are the most peculiar features/feature combinations for the different groups, we plotted the average of the classes using the parallel coordinates paradigm, e.g. for each feature set considered, we plotted a graph with the different variables, rescaled in the range [0 1] on a set of parallel axes and representing each category with a line joining the average values on each axis. We obtain in this way polylines that may be considered sorts of class signatures.
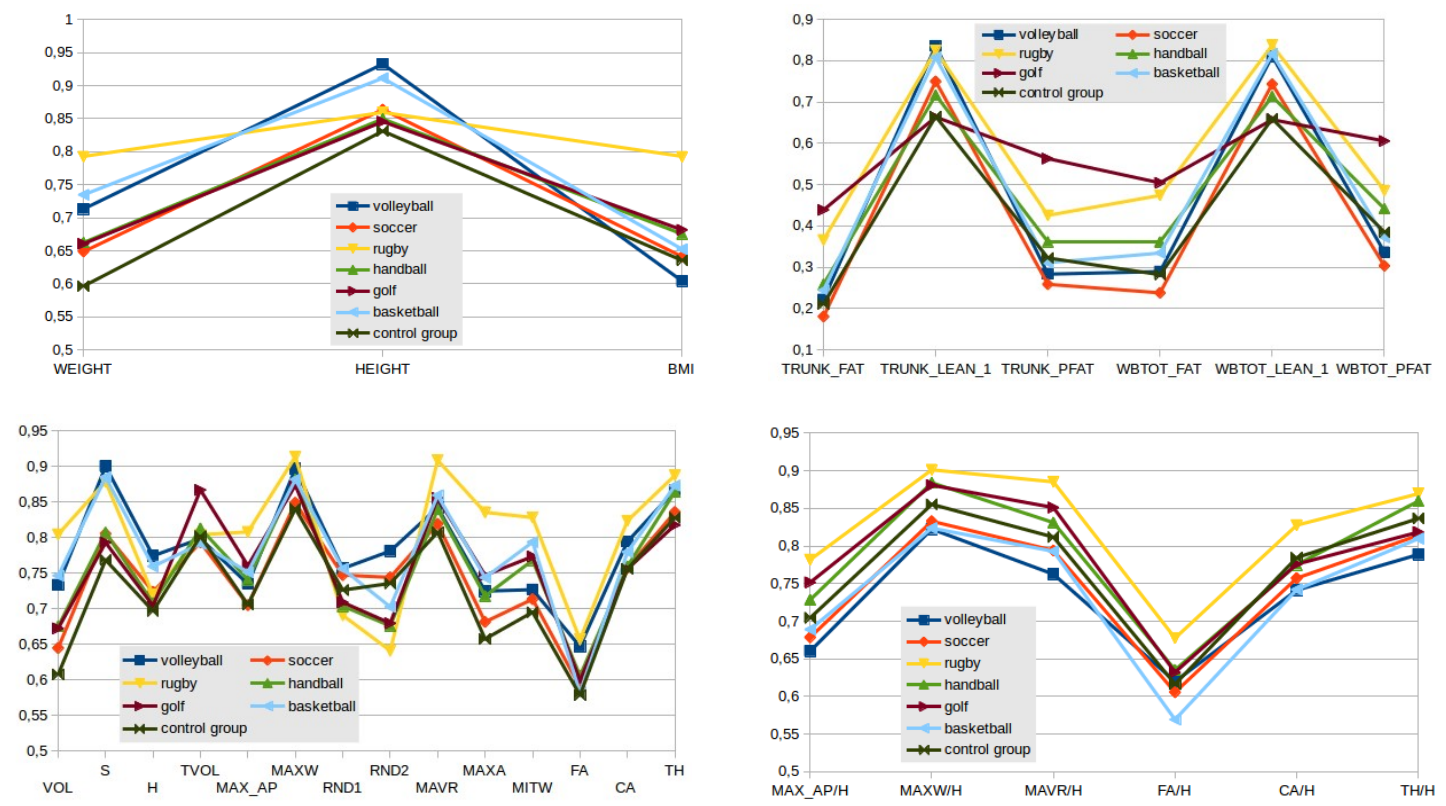

Figure 3: Visualization of average parameters on rescaled ranges for the different classes in a "parallel coordinates" visualization fashion. Top left: manual mesaurements. Top right: DXA values. Bottom left: automatic measurements. Bottom right: selected normalized measurements. 
These plots (Figure 3), show that from weight/height/BMI peculiar features of volley and basketball players (larger height) and rugby players (higher BMI) are evident, but other classes are mixed (top left). From DXA values (top right) it is possible to see differences in rugby players and, as already noted, golf players.

Only from geometric measurements, however, it is possible to see some other differences that can be considered as typical traits of professional athletes. For example it appears that forearm diameter can be a feature differentiation volleyball and basketball players (evident when normalized by height) as well as average radius of trunk area section. Other categories, however, are still quite difficult to be separated, for example handball players seems quite difficult to be characterized.

\section{Automatic classification}

We finally try to evaluate the possibility of classifying athletes by means of the collected features. For this task we performed leave-one-out classification training classifiers with all subjects but one, testing with the left one, and averaging results. First of all we considered the multi-class problem, e.g. labelling each subject with the corresponding sport and trying to automatically infer labels with the trained classifier. This task is quite hard as classes are heavily mixed in the feature spaces as shown in the previous sections.

The leave-one out classification results obtained using linear Bayesian classifiers trained with single groups of measurements (manual, DXA, automatic) are actually quite bad (average error ranging from $44 \%$ to $48 \%$ for the different sets). However, if all the features are used together, the average classification error in the multiclass problem reaches a more reasibable $34 \%$.

Automatic measurements do not improve the accuracy with respect of using weight, height and DXA data. However, selecting the measures that we know to be well correlated with fat values and the limb measurements that should provide independent information and adding these values to weight/height information only we have still an improved error of $39 \%$ meaning that automatic measurements carry independent information differentiating classes.

It may be also interesting to evaluate single class discrimination from the rest. We therefore performed also similar cross-validation tests with labelling of single group against all the other data.

Table 5 presents the classification errors obtained using different features and features combination. It seems that different categories are better characterized by different parameters, e.g. volley and basketball players are better discriminated using measurements, golf players are mostly characterized by DXA values.

\section{Discussion}

We presented an analysis of anthropometric features of professional sports players, using manual and automatically extracted measurements as well as DXA data with an aim at identifying sportspecific characteristics. Knowing which athletes from different sports share similar physical characteristics would be valuable information when directing young athletes towards sports that optimally suit their specific, individual characteristics or to evaluate the effect of specific strength and conditioning programmes. This preliminary work confirms and expands over previous knowledge by showing that simple, traditional anthropometric parameters can reasonably discriminate between different sports and new digital, automatically extracted parameters such as FA and CA may valuably add to the discriminating ability of anthropometry. While some results should be considered with caution due to the low number of athletes involved (e.g., golfers and volleyball players), data clearly suggest that each sport is characterized by athletes with peculiar physical attributes. However, it seems that soccer players are more difficult to discriminate vs. other sports participants (Table 5). This may be due to the wide range of physical characteristics in soccer players according to the playing position. Further investigation in a larger number of players is needed to clarify this issue. Taken together these preliminary results show that advanced imaging techniques such as automatic digital anthropometry is useful to detect sport-specific features over a wide range of sports.

Average LOO classification error (\%)

\begin{tabular}{|l|r|r|r|r|r|r|r|}
\cline { 2 - 8 } \multicolumn{1}{c|}{} & \multicolumn{1}{c|}{ volley vs all } & soccer vs all & rugby vs all & handball vs all & golf vs all & basket vs all & control vs professionals \\
\hline H/W/BMI & 5,6 & 40,7 & 9,4 & $\mathbf{8 , 6}$ & 3,9 & 16,1 & $\mathbf{1 2 , 8}$ \\
\hline DXA & 5,1 & 23,1 & 13,3 & 9,4 & 3 & 19,3 & 15 \\
\hline Measurements & 6,8 & 37,7 & 9 & $\mathbf{8 , 6}$ & 3,8 & 16,3 & 15,1 \\
\hline All & 4,3 & $\mathbf{2 2 , 7}$ & $\mathbf{7 , 2}$ & 10 & 4,2 & $\mathbf{1 0}$ & 13,3 \\
\hline Meas+W/BMI & 4,3 & 40,3 & 8,5 & $\mathbf{8 , 6}$ & 4,2 & 16,3 & 12,8 \\
\hline
\end{tabular}

Table 5: Average Leave One Out classification errors obtained on 2-classes problems (category vs all others) using different features sets Bold font indicates lowest error. 


\section{References}

[1] A.C. Fry, D, Ciroslan, M.D. Fry, C.D. LeRoux,B.K. Schilling, L.Z. Chiu, Anthropometric and performance variables discriminating elite American junior men weightlifters. Journal of Strength and Condioning Reserach, Vol. 20, n.4, 2006, pp.861-866.

[2] M. Leone, G. Lariviere, A.S. Comtois, Discriminant analysis of anthropometric and biomotor variables among elite adolescent female athletes in four sports. Journal of Sports Sciences. Vol.20, n.6, 2002, pp.443-449.

[3] J. Sampaio, S. Ibanez, A Lorenzo, M. Gomez, Discriminative game-related statistics between basketball starters and nonstarters when related to team quality and game outcome. Perceptual and Motor Skills, Vol. 103, n. 2, 2006, pp 486-494.

[4] R.M. Smith, W.L. Spinks, Discriminant analysis of biomechanical differences

[5] A. Giachetti, C. Lovato C. F.Piscitelli, C. Milanese, C. Zancanaro. Robust automatic measurement of 3D scanned models for human body fat estimation. "IEEE JOURNAL OF BIOMEDICAL AND HEALTH INFORMATICS», vol. 19 , n. 2 , 2015 , pp. 660-667

[6] P. Cignoni, M. Callieri, M. Corsini, et al. Meshlab: an open-source mesh processing tool. In Eurographics Italian Chapter Conference, pages 129-136. The Eurographics Association, 2008.

[7] Kruskal, J. B., and Wish, M. (1978), Multidimensional Scaling, Sage University Paper series on Quantitative Application in the Social Sciences, 07-011. Beverly Hills and London: Sage Publications between novice, good and elite rowers. Journal of Sports Sciences, Vol. 13, n.5, 199, pp. 5377-5385. [8] F. Van Der Heijden et al. Classification, parameter estimation and state estimation: an engineering approach using MATLAB. John Wiley \& Sons, 2005. 